\title{
Temperature restriction in entomopathogenic bacteria
}

3 Alexia Hapeshi1 $\diamond$, Joseph R.J. Healey1, Geraldine Mulley2 and Nicholas R.

4 Waterfield $1^{*}$

6 1Microbiology and Infection Unit, Warwick Medical School,

7 The University of Warwick, Gibbet Hill Road, Coventry, CV4 7AL, UK.

8 2School of Biological Sciences, University of Reading, Whiteknights, Reading RG6

$96 \mathrm{AJ}, \mathrm{UK}$.

10 * Correspondence:

11 Corresponding Author

12 n.r.waterfield@warwick.ac.uk

$14 \diamond$ Present address: Chemistry Department, University of Warwick, Gibbet Hill Road, 15 Coventry, CV4 7AL, UK.

Keywords: temperature, adaptation, enterobacteria, Photorhabdus, symbionts, 18 pathogens

\section{Abstract}

Temperature plays an important role in bacteria-host interactions and can be a

23 determining factor for host switching. In this study we sought to investigate the reasons

24 behind growth temperature restriction in the entomopathogenic enterobacterium

25 Photorhabdus. Photorhabdus has a complex dual symbiotic and pathogenic life cycle.

26 The genus consists of 19 species but only one subgroup, previously all classed together

27 as $P$. asymbiotica, have been shown to cause human disease. These clinical isolates 
necessarily need to be able to grow at $37{ }^{\circ} \mathrm{C}$, whilst the remaining species are largely restricted to growth temperatures below $34{ }^{\circ} \mathrm{C}$ and are therefore unable to infect mammalian hosts. Here, we have isolated spontaneous mutant lines of $P$. laumondii DJC that were able to grow up to $36{ }^{\circ} \mathrm{C}-37^{\circ} \mathrm{C}$. Following whole genome sequencing of 29 of these mutants we identified a single gene, encoding a protein with a RecG-like helicase domain, that for the majority of isolates contained single nucleotide polymorphisms. Importantly, provision of the wild-type allele of this gene in trans restored the temperature restriction, confirming the mutations are recessive, and the dominant effect of the protein product of this gene. The gene appears to be part of a short three cistron operon, which we have termed the Temperature Restricting Locus (TRL). Transcription reporter strains revealed that this operon is induced upon the switch from $30{ }^{\circ} \mathrm{C}$ to $37{ }^{\circ} \mathrm{C}$, leading to replication arrest of the bacteria. TRL is absent from all of the human pathogenic species so far examined, although its presence is not uniform in different strains of the $P$. luminescens subgroup. In a wider context, the presence of this gene is not limited to Photorhabdus, being found in phylogenetically diverse proteobacteria. We therefore suggest that this system may play a more fundamental role in temperature restriction in diverse species, relating to as yet cryptic aspects of their ecological niches and life cycle requirements.

\section{Introduction}

Photorhabdus is a Gram-negative bacterium that forms a symbiotic relationship with Heterorhabditis nematodes. The Heterorhabditis-Photorhabdus complex is entomopathogenic with the nematodes infecting insects and releasing the bacteria by regurgitation (Ciche and Ensign 2003). Photorhabdus then sets up a lethal septicaemia, producing an array of toxins and degradative enzymes that combat the immune system and kill the insect. They also elaborate a potent cocktail of antimicrobial compounds to eliminate competition by other microbes in the carcass. The bacteria reproduce using nutrients derived from the insect tissues, reaching very high numbers. The nematodes feed on this bacterial biomass, going through several rounds of hermaphroditic reproduction, before eventually re-establishing the symbiosis with the bacteria (reviewed in (Waterfield, Ciche, and Clarke 2009)). The 
60 Photorhabdus genus, which belongs to the class of $\gamma$-proteobacteria, was thought to

61 consist of three species $P$. asymbiotica, $P$. luminescens and P. temperata. Recently,

62 however, it has been proposed that several of the subspecies be elevated to species

63 level resulting in a total of 19 species (Machado et al. 2018). Nevertheless, for the

64 purposes of this study we will refer to these as belonging to the P. asymbiotica, $P$.

65 luminescens and $P$. temperata subgroups. Thus, so far, only species belonging to the

66 P. asymbiotica subgroup (that can grow up to $37^{\circ} \mathrm{C}$ or above) have been identified as

67 the causative agent of human infections (Gerrard and Stevens 2017). The only

68 exception to this was a strain of $P$. luminescens, identified using $16 \mathrm{~S}$ sequencing,

69 which was associated with cutaneous lesions and bacteremia in a hypothermic infant

70 with a body temperature upon admission of $33.6{ }^{\circ} \mathrm{C}$ (Dutta et al. 2018). No other

71 members of the subgroups $P$. luminescens and $P$. temperata, the majority of which are

72 largely restricted to growth temperatures below $37^{\circ} \mathrm{C}$ (Fischer-Le Saux et al. 1999;

73 Tailliez et al. 2010; Machado et al. 2018), have been reported to cause human disease.

74 The exact reasons behind this discrepancy are unknown.

The establishment and maintenance of symbiosis is also a very important aspect of the Photorhabdus life cycle. There is considerable partner preference in the association between Photorhabdus bacteria and the Heterhorhabditis nematodes, although this is not strictly species-specific (Maher et al. 2017; Kazimierczak et al. 2017). Nonetheless, there is evidence for the co-evolution of the two partners (Maneesakorn et al. 2011). It may therefore be of relevance, that the optimum temperature for the nematodes is also relatively low. For example, in our hands the $P$. laumondii DJC Heterorhabditid nematode partner dies in culture above $34{ }^{\circ} \mathrm{C}$ (unpublished data). normally unable to grow at temperatures above $35^{\circ} \mathrm{C}$ on solid LB media. In this 


\section{Results}

\subsection{Isolation and characterisation of temperature tolerant P. laumondii DJC mutants}

Clones of $P$. laumondii DJC were isolated with the ability to grow on LB agar at 36 ${ }^{\circ} \mathrm{C}$. This was following plating of a saturated overnight culture and incubation of the plates at the higher temperature. The process was repeated two more times and colonies were isolated from all three experiments originating from four independent lineages in total. Isolates were re-streaked 5 times at $36{ }^{\circ} \mathrm{C}$ to select for stably growing clones. Overall, the average frequency with which stable clones were isolated was $2.6 \pm 2.5 \times 10-6$. Thirty of those were then analysed by whole genome sequencing to identify any mutations that may give rise to the phenotype. Sequencing was performed using the MiSeq platform and good quality reads were obtained for 29 of the 30 clones. The WT strain in the lab was also sequenced to account for variations between isolates in different laboratories. Reads from the temperature tolerant clones were then mapped against the reference to identify single nucleotide polymorphisms (SNPs).

Of the 29 isolates successfully sequenced, 19 had a SNP in an uncharacterised gene PluDJC_RSO1875 (Figure 1A and Table 1), and an additional isolate (S15) had an

111 IS30-family transposase insertion near the 3' end (chromosomal position 393017).

112 This gene appears in the automated annotation as "recG", however it should be noted

113 that a full-length orthologue of the bona fide $\operatorname{rec} G$ is encoded elsewhere in the

114 genome. RecG is a 693 amino acid protein whose homologue in other bacteria has

115 been well studied and is known to be important for DNA repair and recombination

116 (Ishioka, Iwasaki, and Shinagawa 1997; Rudolph et al. 2010). As PluDJC_RS01875 is

117 involved in the temperature restriction phenotype, and to distinguish it from the $\operatorname{rec} G$

118 misnomer, we subsequently named it $\operatorname{trl} G$ (temperature restriction locus $\mathbf{G}$ ).

119 Repetition of these experiments aimed at isolating temperature tolerant clones by a 120 collaborator in an independent laboratory (Dr G. Mulley), gave rise to two additional 
121 tolerant isolates, named JA and JB. These were specifically tested for the presence of

122 mutations in $\operatorname{trl} G$ by sequencing of a PCR amplicon. Clone JA was found to contain a

123 SNP, while in clone JB an IS66 transposable element had inserted into the 3' end of

$124 \operatorname{trlG}$ (chromosomal position 393014). Homology and structural models of the TrlG

125 protein suggest it comprises two distinct functional domains connected by a flexible

126 loop region. Most of the mutant SNPs were located in either of the two domains.

127 Additionally, three mutants exhibited premature stop codon mutations in the linker

128 region (Figure 1B). Based on a 3D structure prediction of the protein, we can observe

129 that four of the SNPs would result in substitutions in alpha helices potentially

130 disrupting the structure (Figure 1C). The remaining isolates that did not have a

131 mutation in $\operatorname{trl} G$ were actually unable to resume growth at $36{ }^{\circ} \mathrm{C}$ after being frozen

132 (Figure 2A and Supplementary Figure 1). It should be noted that the tolerant isolates

133 are less pigmented and produce less bioluminescence at the higher temperature

134 (Figure 2A and Supplementary Figure 2). We then investigated the hypothesis that the

135 operon might affect growth or survival at colder temperatures by incubating the

136 bacteria at lower temperatures before shifting back to $28^{\circ} \mathrm{C}$. However, the mutants in

137 this case behaved similarly to the WT bacteria (data not shown).

\subsection{The $\operatorname{trl} G$ gene is part of a small putative three gene operon.}

141 Examination of the genomic context of $\operatorname{trl} G$ revealed it is part of a small operon with

142 PluDJC_RSO1880 and PluDJC_RSO1885, encoded between the DNA polymerase

143 gene, polA, and $d s b A$ (Figure 1A). PluDJC_RSO1880 encodes a protein annotated as a

144 histidinol phosphatase (WP_011144772.1). The product of PluDJC_RSO1885

145 (WP_011144773.1) on the other hand is a small 79 amino acid protein annotated as a

146 hypothetical protein but homologues in other organisms are annotated as transcription

147 factors.

148 To further confirm the role of $t r l G$ in temperature restriction we constructed an

149 unmarked deletion mutant of the entire TRL operon, $\Delta t r l$. This mutant was also able

150 to grow on both solid and liquid media at $36^{\circ} \mathrm{C}$ (Figure 2B). Importantly, plasmid

151 trans-complementation with the whole operon restored the temperature restricted 
152 phenotype in the SNP mutants (Figure 3). Note, we used the whole operon rather than

153 just $\operatorname{trl} G$ alone to ensure the stoichiometry of the three gene members was preserved.

155 Finally, since virulence of the bacteria to insects is a crucial aspect of their life cycle,

156 we determined if mutation of $\operatorname{trl} G$ would have any effect on virulence against

157 Galleria mellonella larvae. The experiment was performed at room temperature ( 20

$158{ }^{\circ} \mathrm{C}$ ), using a representative SNP mutant as well as the $\Delta t r l$ deletion strain, compared

159 to WT and the RifR isogenic parent strains respectively. Interestingly, we observed

160 that the SNP mutant killed the Galleria larvae much faster than the WT (Figure 4).

161 This was also the case for the $\Delta t r l$, albeit the effect was less pronounced. We also

162 performed this virulence assay at $36{ }^{\circ} \mathrm{C}$. As expected the WT strain was not able to

163 kill the Galleria, although surprisingly, even though they could grow at this

164 temperature in vitro, the mutant strains also failed to establish an infection.

\subsection{Transcription of the TRL operon is induced by temperature up-shift}

168 To determine whether the TRL operon is specifically expressed in response to exposure to the higher temperature we created reporter constructs whereby we cloned regions upstream of the operon upstream of a promoterless GFP gene. An in silico search for promoters using BPROM (Solovyev and Salamov 2011) showed the presence of two putative promoters upstream of the first gene in the TRL operon (PluDJC_RS01880, designated here as $t r l R$ ). Interestingly, one of these promoters seems to be upstream of the predicted, oppositely encoded polA promoter. Analysis of apparent transcription start sites in our unpublished RNAseq analysis of $P$. laumondii supports the location of these predicted promoters. Additionally, a putative promoter is predicted which is encoded inside the $t r l R$ open reading frame. With TrlR itself showing homology to a transcriptional regulator we hypothesised that this might be a legitimate promoter driving expression of $t r l F$ and $t r l G$. We therefore decided to

180 create multiple constructs to ensure that we capture the transcriptional elements that 181 control expression of genes in the TRL operon (Figure 5A). These constructs were separately introduced back into a rifampicin resistant isolate of DJC and the effect of 
183

184

185

186

187

188

189

190

temperature up-shift tested. These experiments confirmed that transcription of the operon is increased upon temperature up-shift (Figure 5B and 5C). It should be noted that the growth of $P$. laumondii DJC following temperature up-shift does not immediately cease, but rather continues to increase briefly before reaching a plateau (Supplementary figure 3A). Moreover, at the end of the $24 \mathrm{~h}$ period and following a $\sim 20 \mathrm{~h}$ incubation at the higher temperature, a large proportion of cells remain viable as shown by live/dead staining (Supplementary figure 3B). Importantly, the expression of these reporter constructs when the bacteria are grown at $28{ }^{\circ} \mathrm{C}$ was also monitored and we observed that the operon is also expressed at the permissive growth temperature but expression shows a gradual increase as the bacteria go through late exponential and stationary phase (Figure 5B and 5C).

\subsection{Presence of the TRL in the Photorhabdus genus}

Since mutations in $\operatorname{trlG}$ appear to allow $P$. laumondii to survive in higher temperatures we were interested in investigating the presence of the gene among other Photorhabdus genomes. A trlG homologue was found in 21 out of the 46

Photorhabdus genomes in the RefSeq genome database (Figure 6 and Supplementary

Figure 4). However, no $\operatorname{trl} G$ homologues were present in any bacteria belonging to the $P$. asymbiotica or $P$. temperata subgroups. Within the group of bacteria previously classed as $P$. luminescens the presence of the gene is not uniform. Those isolates that possess the gene actually encode for the full operon. Even though the majority of those genomes are in the draft sequencing stage, we investigated the location of the operon in the genome and observed that it appears to be conserved in the region between $d s b A$ and polA. Importantly, in the published genome sequence of the type strain P. laumondii (Duchaud et al. 2003) an insertion sequence (IS) element is found upstream of the polA gene suggesting the operon may have been horizontally acquired. However, we note that this IS element is absent in our WT P. laumondii DJC strain - which is a laboratory selected derivative of the original TT01 isolate (confirmed by PCR). Interestingly, Alien Hunter analysis (Vernikos and Parkhill 2006) shows no evidence of horizontal gene transfer of this three gene TRL locus, suggesting it is ancestral in this sub-lineage of Photorhabdus. In addition to $P$. 
laumondii TT01, only P. laumondii strain DSPV002N possesses an IS630-like element ISPlu10 family transposase directly upstream of the operon. Four other isolates, P. laumondii HP88, Photorhabdus sp. S7-51, Photorhabdus sp. S15-56 and

218 Photorhabdus sp. S14-60, appear to possess a gene encoding for a 61 amino acid 219 hypothetical protein (WP_133148736.1), upstream of the operon but in the opposite 220 strand.

\subsection{The TRL is conserved amongst bacterial genera}

The TRL is of relevance to bacteria beyond the Photorhabdus genus. Blastp analysis against the NCBI reference protein database showed that TrlG is prevalent in proteobacteria (Figure 7). The closest homologue outside of Photorhabdus is found in Vibrio parahaemolyticus followed by proteins encoded by Pseudospirillum japonicum and Nitrincola nitratireducens, which like Photorhabdus belong to the class of $\gamma$-proteobacteria. TrlG was, however, absent from the closely related Xenorhabdus, also a nematode endosymbiont. While the TrlG proteins of

231 Photorhabdus and Glaeserella are all clustered together, for other bacteria this does 232 not seem to be the case, suggesting individual acquisition events. Within the Vibrio 233 genus, the gene is found in specific isolates of different species, such as $V$. cholerae,

$234 V$. anguillarum and V. atlanticus. As an example, out of the 1218 V. cholerae

235 genomes found in the RefSeq database, only 12 were found to encode for a 236 homologue of TrlG (with over $40 \%$ sequence identity). Of note is the presence of

237 TrlG in various Pseudomonas isolates. Some are classified as strains of the plant 238 pathogens Pseudomonas syringiae or Pseudomonas amygdali, however the gene is 239 also present in some isolates of P. aeruginosa.

241 Analysis of conservation of the entire operon using MultiGeneBlast (Medema,

242 Takano, and Breitling 2013) against a Genbank database consisting of complete 243 bacterial genomes showed that outside of the Photorhabdus genus, all three genes, 244 pluDJC_RS01875 - RS01885 are only conserved syntenically in strains of Vibrio 245 anguillarum and on plasmid pYR3 of Yersinia ruckeri strain CSF007-82 (locus tags 
CSF007_p0360, CSF007_p0365, CSF007_p0345, Figure 8). However, unlike the

247 TRL of $P$. laumondii where the three genes are located in succession, in pYR3 there

248 are two small genes annotated as encoding Type I restriction-modification system,

249 DNA-methyltransferase subunit M in between CSF007_p0345, the gene encoding the

250 putative transcriptional regulator, and CSF007_p0360. Yersinia ruckeri is the

251 causative agent of enteric red mouth disease in salmonid fish species (Kumar et al.

252 2015) and its optimum growth temperature is also $28^{\circ} \mathrm{C}$, while its optimum

253 temperature for infectivity is $18{ }^{\circ} \mathrm{C}$. Similarly, $V$. anguillarum is also a fish pathogen

254 with an optimum growth temperature of $25^{\circ} \mathrm{C}$ whilst the optimum temperature for

255 vibriosis disease is $15{ }^{\circ} \mathrm{C}$ (Lages, Balado, and Lemos 2019). It appears that a lower

256 temperature optimum for virulence is common in fish pathogens and virulence genes

257 are found to be up-regulated at these lower temperatures (Guijarro et al. 2015;

258 Mendez et al. 2018; Lages, Balado, and Lemos 2019).

\section{Discussion}

Members of the genus Photorhabdus dedicate a significant proportion of their

263 genome to the synthesis of virulence factors and resource defence. Indeed, when the

264 first Photorhabdus genome sequence was completed it was noted that there were

265 more predicted toxin genes than in any other bacteria that had been sequenced at the

266 time (Duchaud et al. 2003). In addition, many other genera belonging to the $\gamma$ -

267 proteobacteria contain multiple examples of mammalian pathogens, both obligate and

268 opportunistic. With these observations in mind it is perhaps unusual that only very

269 few members of the genus Photorhabdus are able to cause infection in humans

270 (Gerrard and Stevens 2017). Our previous analysis of the adaptations relevant to

271 human pathogenicity in a representative $P$. asymbiotica strain suggested that no

272 specific mammalian active toxins are necessary to facilitate human infection. Rather it

273 seems that substantive changes in metabolic activity, when cultured at $37^{\circ} \mathrm{C}$, are

274 primarily responsible for their expanded host range (Mulley et al. 2015); a

275 phenomenon termed nutritional virulence (Abu Kwaik and Bumann 2013). 
277 An obvious limitation for mammalian infection in many members of the genus is their

278 inability to grow above $\sim 35^{\circ} \mathrm{C}$, which again is unusual for members of the $\gamma$ -

279 proteobacteria. Typically, maximum growth temperatures of bacteria are determined

280 by multiple factors that affect bacterial physiology, such as membrane and lipid

281 fluidity, chaperone activities and other enzyme/protein functions. It is therefore

282 possible that for any one species there are multiple functions that fail simultaneously

283 at the non-permissive temperature. However, the ability of $P$. asymbiotica isolates to

284 grow readily at temperatures of $37^{\circ} \mathrm{C}$ (and above) and the overall high level of

285 conservation in core genome sequence across the genus indicates that there is likely

286 no general physiological reason for temperature restriction in members of the genus.

288 Our identification of the TRL genes offers up an alternative explanation to this 289 temperature restriction phenomenon, at least in certain lineages of $P$. laumondii. The

290 specific induction of the TRL gene transcription upon temperature up-shift, leading to 291 growth arrest, appears prima facia to represent a specific adaptive mechanism. We do 292 not yet know if induction of TRL transcription at higher temperatures is mediated by 293 the TrlR protein alone or if other regulatory pathways are involved. While it remains 294 unclear as to what advantage this temperature dependent growth arrest imparts upon 295 these strains, we may speculate. For example, we note that the nematode symbiont 296 cannot survive above $\sim 34{ }^{\circ} \mathrm{C}$ in vitro, although to our knowledge nematode survival 297 at different temperatures in vivo has not been investigated. Therefore, a TRL mediated 298 mechanism to suppress continued exponential growth at increasing temperatures, may 299 represent a mechanism to synchronise life cycles of the bacteria and nematode in 300 fluctuating environmental conditions. It is perhaps no coincidence that the TRL 301 operon is encoded next to the polA gene and that the most 5' TRL promoter overlaps 302 on the opposing strand with that of the polA gene. Thus, activation of TRL 303 transcription might directly suppress polA transcription by steric hindrance. It is 304 formally possible that the tight linkage between polA and TRL reflects a functional 305 linkage in the gene activities (ie, replication and temperature). It is interesting in this 306 respect that even at $28{ }^{\circ} \mathrm{C}$, we see a gradual increase in TRL transcription as the 307 culture moves toward stationary phase and the eventual cessation of replication. 
309 Interestingly, while ablation of the whole TRL operon did not strongly affect

310 virulence in the Galleria infection model at $28^{\circ} \mathrm{C}$, the S1 SNP mutant actually

311 became significantly more virulent at this temperature. This suggests a functional

312 interplay between $\operatorname{trl} G, \operatorname{trlF}$ and $t r l R$, as only the $\operatorname{trl} G$ gene is mutated in $\mathrm{S} 1$.

313 Therefore, the activity of $\operatorname{trlF}$ and/or $\operatorname{trlR}$ in the absence of the balancing effect of $\operatorname{trl} G$

314 seems to enhance virulence activity. Importantly, when the host temperature was

315 increased to $36{ }^{\circ} \mathrm{C}$, the infection failed of both the S1 SNP and $\Delta t r l$ strains, despite

316 their ability to grow in vitro. This is consistent with the reported failure of $P$.

317 asymbiotica to establish a lethal infection in the Manduca sexta model when

318 incubated at $37^{\circ} \mathrm{C}$ (Mulley et al. 2015). In a natural context $37^{\circ} \mathrm{C}$ is not a problem

319 for P. asymbiotica ATCC43949 as it would be in a mammalian host, for which it is

320 adapted. Thus, despite the alleviation of growth restriction, there are clearly other

321 temperature dependent changes in gene regulation occurring, as evidenced by the

322 down regulation of luciferase activity and pigment production in these mutants. This

323 effect may be common to both $P$. asymbiotica and the mutant $P$. laumondii lineages

324 and regulated through a separate unknown mechanism. In addition, the loss of

325 pigmentation and reduction in bioluminescence of the mutant colonies at $36{ }^{\circ} \mathrm{C}$ is

326 reminiscent of the secondary phenotypic variants of Photorhabdus (Boemare and

327 Akhurst 1988) as well as $\Delta m d h$ and $\Delta f u m C$ mutants deficient in enzymes of the TCA

328 cycle (Lango and Clarke 2010) and a $\Delta$ relAspoT strain, deficient in (p)pGpp synthesis

329 (Bager et al. 2016). The aforementioned mutants retain virulence in insect models but

330 are no longer symbiotically competent. Absence of pigmentation and

331 bioluminescence is also a characteristic of the M-phase variants of $P$. luminescens,

332 which are responsible for transmission of the bacteria to infective juvenile nematodes

333 and establishment of symbiosis, however those variants are avirulent against insects

334 (Somvanshi et al. 2012).

336 The majority of random mutations selected from the population were non-

337 synonymous amino acid substitutions in TrlG rather than premature stop codons or

338 frame shifts. It is possible that this simply reflects the lower probability of obtaining a

339 stop codon by random chance as complete deletion of the operon suggested the

340 presence of an altered version of $\mathrm{TrlG}$ was not in some way advantageous. More 
341 importantly, no mutations were seen in the much larger and tightly linked $t r l F$ gene.

342 This suggests that either (i) TrlF does not play a role in the temperature restriction

343 phenotype, or (ii) that if it does, its function can be fully compensated by dominant

344 activity of an intact $\mathrm{Tr} l \mathrm{G}$ protein. The small size of the $\operatorname{trl} R$ regulator gene means

345 there was a much lower probability of isolating mutants in this. As we could delete

346 the whole operon, it indicates that mutations in $t r l F$ alone are not overtly deleterious if

347 they should occur in isolation.

348 As the lifestyles of most Photorhabdus strains are generally similar, it is interesting 349 that the TRL operon appears restricted yet ancestral to a specific species lineage of 350 Photorhabdus. As members of this linage are geographically dispersed (Machado et 351 al. 2018; Mathur et al. 2018), factors such as biogeography, insect prey type or the 352 nature of competing organisms are unlikely to be the reason for maintaining TRL. We 353 therefore speculate that selection for TRL may be a result of specific requirements of 354 the host bacterial genomes, or perhaps in restrictions imposed by the nematode host 355 lineage. For example; as the $P$. luminescens TT01 genome is heavily infested by IS356 elements, we could hypothesise that TRL represents a mechanism to protect against 357 rampant IS-element activity. If a sustained temperature increase was "perceived" as 358 stress to the bacteria (or more importantly the IS-elements), it may lead to undesirable 359 activation of a large number of IS-elements. Therefore, stopping genome replication 360 would likely protect against extensive transposition damage.

An alternative idea is that these strains rely on the activities of a pathway or global

363 regulator which is highly sensitive to temperature. For example, it has been shown 364 that secondary metabolite production is reliant upon the activity of the Hfq protein 365 (Tobias et al. 2017) which, in other Gram-negative bacteria studied, mediates 366 interactions of regulatory ncRNAs with their target mRNA molecules (A. Zhang et al. 367 1998; Møller et al. 2002; Lease and Woodson 2004). Folding and interactions of 368 many ncRNAs are often temperature sensitive. The lack of pigment and 369 bioluminescence in the mutant strains at the higher temperature suggests that this 370 regulation is indeed being perturbed. If all secondary metabolism necessary for 371 virulence and symbiosis ceases at the higher temperature it would make sense for the 372 bacterium to inhibit further replication until normal regulation is re-established. 
374 Finally, the identification of TRL-like operons in members of other genera suggests

375 this represents a widespread general mechanism, regulating the life-cycles of

376 phylogenetically diverse bacteria. The association of TRL operons with pathogens of

377 poikilothermic hosts (e.g. fish) tempts speculation there is an as yet unknown

378 advantage to this mechanism in such niches.

\subsection{Strains and growth conditions}

383 The strains used in this study were the rifampicin sensitive parent strain of $P$.

384 laumondii subsp. laumondii DJC (WT) (Zamora-Lagos et al. 2018), and a rifampicin 385 resistant derivative of this strain, P. luminescens spbsp. laumondii DJC (RifR) (Jones 386 et al. 2010). Bacteria were grown in Lysogeny broth (LB) at $28^{\circ} \mathrm{C}$ unless otherwise 387 stated. For growth on solid media, LB was supplemented with $1.5 \%$ agar and $0.1 \%$ 388 sodium pyruvate and plates were incubated in the dark. When necessary, kanamycin 389 was added to the media at a concentration of $25 \mu \mathrm{g} / \mathrm{ml}$. To assess growth on solid 390 media, overnight cultures of Photorhabdus were diluted to an OD600 of 0.05 and $5 \mu \mathrm{l}$ 391 were spotted on solid media. They were then incubated at the appropriate temperature 392 for $48 \mathrm{~h}$. To assess growth in liquid, the diluted cultures were incubated at the 393 appropriate temperature for $24 \mathrm{~h}$, shaking at $180 \mathrm{rpm}$.

\subsection{Isolation of the temperature tolerant clones}

396 Saturated overnight cultures of $P$. laumondii DJC grown at $28^{\circ} \mathrm{C}$ in LB medium were 397 plated on LB agar (LBA) and incubated at $36^{\circ} \mathrm{C}$. After approximately $48 \mathrm{~h}$ colonies 398 were observed on the plates. These were re-streaked 4 times on LBA at $36{ }^{\circ} \mathrm{C}$ and 399 stably grown clones were stored at $-80{ }^{\circ} \mathrm{C}$ in $32 \% \mathrm{v} / \mathrm{v}$ glycerol. 
401

402

403

404

405

406

407

408

409

410

411

412

413

414

415

416

417

418

419

420

421

422

423

424

425

426

\subsection{Whole-genome sequencing and bioinformatics analysis}

DNA was isolated from $1 \mathrm{ml}$ of overnight cultures of $P$. laumondii DJC using the Qiagen Blood and Tissue kit. The Nextera XT kit (Illumina) was used for library preparation and sequencing was performed using a MiSeq v2 cartridge on a MiSeq sequencer (Illumina). Raw data has been deposited in the NCBI database under BioProject ID PRJNA596169, while raw sequencing reads for the WT isolate in our lab can be found in the short read archive using accession number SRR8307122. Fastq reads of both the WT and the tolerant clones were mapped against the NCBI reference sequence for $P$. luminescence subsp laumondii DJC

NZ_CP024900.1(Langmead and Salzberg 2012) in very sensitive mode. SAMtools v0.1.18 ( $\mathrm{Li}$ et al. 2009) was used to obtain sorted bam files, containing aligned reads, and subsequently pile up format files. VarScan v2.3.6 (Koboldt et al. 2012) was then used to identify SNPs (parameters: p-value 0.1, min-coverage 20 --min-reads2 15 -min-avg-qual 30 --min-var-freq 0.9). A different set of parameters was subsequently used to account for the clones with lower coverage (with modified min-coverage of 10 and --min-reads 2 of 6). For clones where no SNP was identified in the $\operatorname{trl} G$ gene automatically, manual inspection of the alignment files followed by PCR and Sanger sequencing were used to check for the presence of any insertions/deletions. In particular, to sequence the $\operatorname{trl} G$ gene in the JC and JD strains as well as the tolerant clones S15 and S29, either the gene was amplified using primer pair 00323SecF/00323SecR or the entire operon was amplified using recOSeqF/reOSeqR and the resulting PCR products were purified and sent for Sanger sequencing. All primer sequences used in this study are listed in Table 2.

A prediction of the protein structure of TrlG was performed using I-TASSER (Y. Zhang 2008) with default parameters. The location of the amino acid substitutions on the protein was visualised using UCSF Chimera (Pettersen et al. 2004).

To investigate the presence of the gene in other bacteria, $t r l G$ was translated using the standard codon usage and the amino acid sequence was used as input for BLASTP v2.8.1 against the NCBI protein reference sequences using default search parameters. Hits were retained if the showed at least $60 \%$ sequence identity over $90 \%$ of the query length. Multiple alignments were created using Clustal Omega (Sievers et al. 
433 2011). The Clustal alignment file was then used to infer a maximum likelihood

434 phylogeny using IQ-TREE v 1.6.3 (Nguyen et al. 2015) with the JTT substitution

435 model selected by ModelFinder (Kalyaanamoorthy et al. 2017), and ultrafast

436 bootstrap (Hoang et al. 2018). To reconstruct the Photorhabdus recA phylogeny, recA

437 nucleotide sequences were obtained from the Photorhabdus genomes present in the

438 NCBI reference genomic sequence database and analysed using the Phylogeny.fr

439 platform. The sequences were aligned using MUSCLE and ambiguity was removed

440 using Gblocks. A maximum likelihood tree was then constructed using PhyML and

441500 bootstrap iterations were performed. Phylogenetic trees were visualised using the

442 Interactive Tree Of Life (iTOL) (Letunic and Bork 2019). For MultiGeneBlast

443 analysis (Medema, Takano, and Breitling 2013), a database was created from the

444 bacterial Genbank division and a homology search was performed with default

445 parameters, with the three TRL genes as input.

\subsection{Construction of a $\Delta t r l$ mutant}

448 A markerless $\Delta t r l$ deletion mutant was created by double homologous recombination 449 using the suicide plasmid pDS132 (Philippe et al. 2004). Regions upstream and 450 downstream of the TRL were amplified using primer pairs DrecOUF/DrecOUR and 451 DrecODF/DrecODR respectively. The purified PCR products were mixed and used as 452 a template in a second joining PCR with primers DrecOUF/DrecODR. The resulting 453 product was digested using enzymes SacI and PstI and ligated into digested and 454 dephosphorylated pDS132. The resulting plasmid was introduced by transformation 455 into the donor E. coli strain S17.1 lamda-pir. For conjugations into Photorhabdus, the 456 rifampicin resistant $P$. luminescens DJC strain was used as a recipient. Briefly, 457 exponentially growing cultures of $P$. laumondii DJC RifR in LB containing $1 \mathrm{mM}$ $458 \mathrm{MgCl}_{2}$ and $E$. coli $\mathrm{S} 17.1$ lamda-pir harbouring $\mathrm{pDS} 132(\Delta t r l)$ were harvested and 459 combined at a ratio 4:1 of acceptor to donor bacteria. Matings were spotted on LBA 460 plates containing $1 \mathrm{mM} \mathrm{MgCl} 2$ and incubated overnight at $28{ }^{\circ} \mathrm{C}$. Bacteria were then 461 harvested and aliquots were plated on LBA supplemented with $15 \mu \mathrm{g} / \mathrm{ml}$ 462 chloramphenicol and $50 \mu \mathrm{g} / \mathrm{ml}$ rifampicin. Possible transconjugants and hence first 463 recombinants were confirmed by PCR using primer pairs TestRecO1/00323SeqR and 464 polA3F'/ TestRecG4. Successful first recombinants were grown in LB overnight and 
465

466

467

468

469

470

471

472

473

474

475

476

477

478

479

480

481

482

483

484

485

486

487

488

489

490

491

492

493

494

495

then the culture was diluted in fresh LB and grown to mid exponential. At this point serial dilutions were made and aliquots were plated on LBA supplemented with $0.2 \%$ sucrose and incubated at $28{ }^{\circ} \mathrm{C}$. The resulting colonies were tested for deletion of the TRL operon by PCR using primers TestRecO1/TestRecG4.

\subsection{Complementation}

For complementation, the entire TRL was amplified using primers recOFEcoRI/recORSpeI and the resulting PCR product was digested with EcoRI and SpeI and ligated into a modified version of vector pBCSK (that had been previously digested with AseI and KpnI to remove Plac). The resulting plasmid, termed pBCSK'(TRL), and the empty pBCSK' were introduced separately into the $\Delta t r l$ deletion mutant or into SNP mutants by transformation. To prepare cells for transformation, bacteria growing exponentially in $100 \mathrm{ml} \mathrm{LB}$ were harvested at an OD600 of 0.2-0.3 and kept for 90 min on ice. They were then collected by centrifugation and washed twice with ice-cold SH buffer at pH 7 (10 mM HEPES containing $5 \% \mathrm{w} / \mathrm{v}$ sucrose). The cells were resuspended in $160 \mu \mathrm{l} \mathrm{SH}$ buffer and 50 $\mu \mathrm{l}$ aliquots were used for electroporation with the following conditions: $2.5 \mathrm{kV}, 25 \mu \mathrm{F}$ and $200 \Omega$ in $2 \mathrm{~mm}$ cuvettes. This was followed by addition of $1 \mathrm{ml} \mathrm{LB}$ and incubation at $28^{\circ} \mathrm{C}, 180 \mathrm{rpm}$ for $3 \mathrm{~h}$. Aliquots were plated on LB agar supplemented with $25 \mu \mathrm{g} / \mathrm{ml}$ chloramphenicol. Colonies were tested for the presence of the plasmid by PCR using primers M13R/Seq03223F.

\subsection{Infection assays}

The infections were performed using 5th instar larvae of Galleria mellonella. The bacteria were grown in LB at $28{ }^{\circ} \mathrm{C}$ and then collected and resuspended in PBS to an OD600 of 1.5. The suspension was then serially diluted to obtain a final suspension with an infection dose of $\sim 50$ colony forming units per $10 \mu \mathrm{l}$. The Galleria larvae were infected by injection through the first proleg and incubated either at room temperature or $36^{\circ} \mathrm{C}$. For the experiment conducted at room temperature two groups of ten larvae were infected per strain, while for the experiment at $36{ }^{\circ} \mathrm{C}, 8$ larvae were infected per strain. The experiment was conducted twice. 


\subsection{Construction of GFP promoter fusions}

498 The TRL promoter regions were amplified using Q5 DNA polymerase and with $P$.

499 laumondii DJC DNA as a template and using the following primer pairs:

500 Prec2F/PrecR for promoter region PA, Prec2F/Prev00325 for promoter region PB,

501 Prec1f/Prev00325 for promoter region PC and Prec3F/Prev00325 for promoter region

502 PD. PCR products were digested using BamHI and SalI and purified. They were then

503 ligated into the broad range vector pGA-G1, which carries a promoterless $g f p$-mut3

504 (Leo Eberl, University of Zurich). The ligations were introduced by transformation

505 into E. coli DH5a lamda-pir. Successful transformants were confirmed by PCR and

506 plasmids were isolated using the Qiagen miniprep kit and introduced into E. coli

507 strain S17.1 lamda pir to be used as a donor in conjugation, which was performed as 508 described above.

\subsection{Growth and fluorescence measurements of reporter strains}

$511 P$. laumondii DJC strains harbouring the reporter constructs were grown overnight in

512 LB supplemented with kanamycin at $28^{\circ} \mathrm{C}$ and the cultures were then diluted to fresh

513 media to an OD600 of $\sim 0.05$. The diluted cultures were aliquoted in black flat-

514 bottomed $\mu$ Clear 96-well plates (CELLSTAR, Greiner) and placed in an Omega

515 Fluostar (BMG Labtech) microplate reader. They were incubated at $28{ }^{\circ} \mathrm{C}$ with double

516 orbital shaking at $300 \mathrm{rpm}$. Readings of absorbance at $600 \mathrm{~nm}$ as well as GFP

517 fluorescence (gain 1000) were taken every $30 \mathrm{~min}$. For the temperature shift

518 experiment, after $5 \mathrm{~h}$ of growth at $28^{\circ} \mathrm{C}$ the temperature of the microplate reader was

519 increased to $36{ }^{\circ} \mathrm{C}$ (this took place in $\sim 2 \mathrm{~min}$ ). Then readings were taken immediately

520 following the shift and at $30 \mathrm{~min}$ intervals after that.

523 We would like to thanks Prof. Mark Pallen for the use of his MiSeq machine. 


\section{Conflict of Interest}

526 The authors declare that the research was conducted in the absence of any commercial or financial relationships that could be construed as a potential conflict of interest.

\section{$7 \quad$ Author Contributions}

$530 \mathrm{AH}$ and NW designed the study; $\mathrm{AH}, \mathrm{JH}$ and GM performed the mutant isolation

531 experiments; JH performed the structural prediction studies; AH performed the

532 remaining experiments and analysed the data. AH and NW wrote the initial

533 manuscript draft. GM reviewed the manuscript. All authors have approved the final

534 manuscript.

\section{$536 \quad 8 \quad$ Funding}

537 This work was funded by Warwick Medical School, University of Warwick, the

538 Leverhulme Trust grant RPG-2015-194 and the EPSRC MOAC Centre for Doctoral 539 Training (DTPEP/F500378/1).

542 Table 1 TrlG amino acid substitutions that result from the SNPs identified in the

543 sequenced isolates, as well as isolates JA and JB. The isolates are separated based on

544 the experiment and thus the lineage they originate from. Note that in experiment 3

545 two independent starting cultures were used. *Inspection of the aligned sequencing

546 reads for clone S29 revealed possible SNPs or small insertions/deletions at the 3' of

$547 \operatorname{trlG}$, however it was not possible to confirm that with Sanger sequencing of the

548 amplified region.

\section{Experiment Clone}

(lineage) TrlG Amino acid substitution 


\begin{tabular}{|c|c|c|}
\hline & $\mathrm{S} 1$ & Ser16 --> Asn \\
\hline & $\mathrm{S} 2$ & $\mathrm{n} / \mathrm{a}$ \\
\hline & $\mathrm{S} 3$ & $\mathrm{n} / \mathrm{a}$ \\
\hline & S4 & Arg126 --> Gln \\
\hline 1 & S5 & $\mathrm{n} / \mathrm{a}$ \\
\hline & S6 & Arg80 --> His \\
\hline & S7 & Arg80 --> His \\
\hline & S8 & $\mathrm{n} / \mathrm{a}$ \\
\hline & S9 & $\mathrm{n} / \mathrm{a}$ \\
\hline & $\mathrm{S} 10$ & $\mathrm{n} / \mathrm{a}$ \\
\hline & $\mathrm{S} 11$ & Ser208 --> STOP \\
\hline & $\mathrm{S} 12$ & Ala193 --> Asp \\
\hline & $\mathrm{S} 13$ & Gly354 --> Val \\
\hline & $\mathrm{S} 14$ & Ala193 --> Asp \\
\hline 2 & $\mathrm{~S} 15$ & $\mathrm{n} / \mathrm{a}$ (IS transposase insertion) \\
\hline & S16 & Gly354 --> Val \\
\hline & $\mathrm{S} 17$ & Gly354 --> Val \\
\hline & $\mathrm{S} 18$ & Ser331 --> Tyr \\
\hline & S19 & $\mathrm{n} / \mathrm{a}$ \\
\hline & $\mathrm{S} 20$ & Ser331--> Tyr \\
\hline & $\mathrm{S} 21$ & $\mathrm{n} / \mathrm{a}$ \\
\hline
\end{tabular}




\begin{tabular}{|c|c|c|}
\hline & S22 & Ala75 --> Thr \\
\hline \multirow[t]{5}{*}{ 3(a) } & S23 & Val35 --> Ile \\
\hline & S24 & Pro318--> Arg \\
\hline & S25 & Ala36 --> Val \\
\hline & S26 & $\mathrm{n} / \mathrm{a}$ \\
\hline & S27 & Asn335 --> Asp \\
\hline \multirow[t]{5}{*}{ 3(b) } & S28 & Gln249 --> STOP \\
\hline & S29 & $\mathrm{n} / \mathrm{a}^{*}$ \\
\hline & S30 & Gln249 --> STOP \\
\hline & JA & Pro318--> Leu \\
\hline & JB & $\mathrm{n} / \mathrm{a}$ (IS transposase insertion) \\
\hline
\end{tabular}

551 Table 2: Oligonucleotide primers used in this study. The positions of restriction sites are shown underlined.

\begin{tabular}{ll}
\hline Name & Sequence (5'-3') and restriction enzyme site \\
\hline polA 3'F & gggattgtctgctatctgag \\
dsbA 3'F & ggattatgatgctgctatgag \\
DrecOUF & aatgagctcgcacgataaggtatgaggag, SacI \\
DrecOUR & gaagtgatttggagtcacatatttaacactactc \\
DrecODF & gagtagtgttaaatatgtgactccaaatcacttc
\end{tabular}




$\begin{array}{ll}\text { DrecODR } & \text { ttactgcagattgaatcgccggtagc, PstI } \\ \text { TestRecO1 } & \text { atacaccatacatggctcc } \\ \text { 00323SeqF } & \text { tggaaggtggtgcagaag } \\ \text { 00323SeqR } & \text { gcgacagtctgattgctc } \\ \text { TestRecG4 } & \text { ttatggcatttagcgtatctg } \\ \text { Prec1F } & \text { ataggatccactgtcatattgccttatag, BamHI } \\ \text { Prec2F } & \\ \text { PrecR } & \text { accggatccaaatcttcttcattatctg, BamHI } \\ & \\ & \\ \text { ttagtcgacattaacactactcacgtgc, SalI } & \end{array}$

\section{$553 \quad 10 \quad$ Figure Legends}

555 Figure 1. A) Genetic organisation of the Temperature restriction locus. B) Domain

556 architecture of TrlG and the presence of substitution mutations in the temperature

557 tolerant clones. Vertical lines indicate the positions of the mutations; red depicts stop

558 mutations. The coloured boxes show the domains identified by Pfam; Magenta:

559 Alba_2 (Pfam domain PF04326, at amino acid positions 14-128) and light blue:

560 HATPase_c_4 (Pfam domain PF13749, at amino acid positions 298-365). C) (Left) I-

561 TASSER simulation of TrlG with the distribution of mutations mapped. Orange

562 indicates SNP positions, whilst red depicts stop mutations. (Right) The same model

563 as in (Left) with homology domains mapped in colour. Images prepared with UCSF

564 Chimera. 
566 Figure 2. A) Growth of the 30 sequenced tolerant clones and P. laumondii DJC (WT)

567 on LB agar at $28^{\circ} \mathrm{C}$ and $36^{\circ} \mathrm{C}$. B) Growth of the additional two clones isolated

568 independently (JA and JB) compared to P. laumondii DJC (WT) as well as of the $\Delta \operatorname{trl}$

569 deletion strain compared to the isogenic rifampicin resistant $P$. laumondii DJC

570 (DJCRif) at $28{ }^{\circ} \mathrm{C}$ and $36^{\circ} \mathrm{C}$.

572 Figure 3. Growth of the $\Delta t r l$ deletion mutant complemented with either the empty

573 pBCSK' vector or pBCSK' carrying the TRL operon on A) LB agar and B) liquid LB

574 media at $28{ }^{\circ} \mathrm{C}$ and $36{ }^{\circ} \mathrm{C}$. The asterisk denotes significant difference at $p_{\text {adj }}<0.05$.

575 Results show the averages and standard error from four biological replicates.

577 Figure 4. Virulence of the $t r l$ mutants. The results show the number of surviving

578 Galleria mellonella larvae infected with either the WT strain, the S1 SNP mutant, the

579 RifR WT strain or the $\Delta \operatorname{trl}$ deletion mutant at different time points. G. mellonella

580 larvae injected with PBS were used as control. Twenty larvae were used for each

581 strain and the experiment was repeated with similar results.

583 Figure 5. Up-regulation of the promoter of the TRL operon upon shift to $36{ }^{\circ} \mathrm{C}$. A)

584 Schematic diagram showing the different regions used in the promoter reporter

585 constructs. The expanded panel shows the region between $t r l F$ (PluDJC_RSO1880)

586 and polA with the predicted promoters based on BPROM indicated as arrows on top

587 of the sequence. At the bottom are the regions amplified and introduced separately

588 into pGAG1 upstream of the gfpmut3 gene to create promoter constructs PAGFP-

589 PDGFP. The diagrams are color coded to reflect the colors of the data plotted below.

590 B) Left: Fluorescence of the $P$. laumondii DJC reporter strains following a shift in

591 temperature from $28{ }^{\circ} \mathrm{C}$ to $36{ }^{\circ} \mathrm{C}$ after $5 \mathrm{~h}$ of growth as indicated by the arrow. Right:

592 Fluorescence of the $P$. laumondii DJC reporter strains grown continuously at $28{ }^{\circ} \mathrm{C}$.

593 Results are arbitrary fluorescence units following a subtraction of the fluorescence of 
594

595

596

597

598

600

601

602

603

604

605

606

607

608

609

610

611

612

613 Figure 8. Conservation and genetic organization of the TRL operon in representative

614 species as detected by Multigene blast. In red is $t r l G$, in green is $t r l F$ and in blue is

$615 \operatorname{trlR}$; genes coloured identically in other organisms denote the identified homologues

616 of $\operatorname{trl} G, \operatorname{trlF}$ and $\operatorname{trl} R$ respectively.

the $P$. laumondii DJC carrying pGAG1 in the absence of any promoter to account for any autofluorescence and they represent the mean of three biological replicates $+/$ standard error. C)The rate of change in fluorescence in P. laumondii DJC carrying pGAG1(PCGFP), calculated by subtracting the fluorescence at a given time point by the fluorescence of the previous time point; left: following a shift in temperature from $28{ }^{\circ} \mathrm{C}$ to $36{ }^{\circ} \mathrm{C}$ after $5 \mathrm{~h}$ of growth as indicated by the arrow; right: at $28{ }^{\circ} \mathrm{C}$. Results represent the mean of three biological replicates $+/$ - standard error.

Figure 6. Maximum likelihood tree constructed using the Photorhabdus recA nucleotide sequences from RefSeq genomes. Black circles indicate branch support over $80 \%$. Highlighted are the strains that were found to contain a copy of the $\operatorname{trl} G$ gene.

Figure 7. Maximum likelihood tree constructed using protein sequences with over 60 $\%$ sequence identity to the Photorhabdus laumondii TrlG protein (with a query cover $>90 \%$ ). Nodes with support values higher than $80 \%$ are indicated with black circles. The external coloured ring represents different genera. Protein identifiers for all the entries can be found in Supplementary table 1.

619 Supplementary Figures 1-4

620 Supplementary Table 1 
622

623

624

625

626

627

628

629

630

631

632

633

634

635

636

637

638

640

641

642

643

644

645

646

647

648

649

650

\section{Data Availability Statement}

The raw sequencing data generated and analysed in this study has been deposited in the NCBI database under BioProject ID PRJNA596169, while raw sequencing reads for the WT isolate in our lab can be found in the short-read archive (SRA) using accession number SRR8307122. The reference genome sequence for P. luminescence subsp laumondii DJC is available on NCBI RefSeq with the accession number NZ_CP024900.1.

\section{References}

Abu Kwaik, Yousef, and Dirk Bumann. 2013. "Microbial Quest for Food in Vivo: 'Nutritional Virulence' as an Emerging Paradigm." Cellular Microbiology 15 (6): 882-90. https://doi.org/10.1111/cmi.12138.

Bager, Ragnhild, Mohammad Roghanian, Kenn Gerdes, and David J. Clarke. 2016. “Alarmone (p)PpGpp Regulates the Transition from Pathogenicity to Mutualism in Photorhabdus Luminescens." Molecular Microbiology 100 (4): 735-47. https://doi.org/10.1111/mmi.13345.

Boemare, Noel, and Raymond Akhurst. 1988. "Biochemical and Physiological Characterization of Colony Form Variants in Xenorhabdus Spp. (Enterobacteriaceae)." Microbiology 134 (3): 751-61. https://doi.org/10.1099/00221287-134-3-751.

Ciche, Todd A., and Jerald C. Ensign. 2003. "For the Insect Pathogen Photorhabdus Luminescens, Which End of a Nematode Is Out?” Applied and Environmental Microbiology 69 (4): 1890-97. https://doi.org/10.1128/AEM.69.4.18901897.2003.

Duchaud, Eric, Christophe Rusniok, Lionel Frangeul, Carmen Buchrieser, Alain Givaudan, Séad Taourit, Stéphanie Bocs, et al. 2003. "The Genome Sequence of the Entomopathogenic Bacterium Photorhabdus Luminescens.” Nature Biotechnology 21 (11): 1307-13. https://doi.org/10.1038/nbt886. 
651 Dutta, Ankhi, Anthony R. Flores, Paula A. Revell, and Lisa Owens. 2018. "Neonatal

652

653

654

655

656

657

658

659

660

661

662

663

664

665

666

667

668

669

670

671

672

673

674

675

676

677

678

679

680

Bacteremia and Cutaneous Lesions Caused by Photorhabdus Luminescens: A Rare Gram-Negative Bioluminescent Bacterium." Journal of the Pediatric Infectious Diseases Society 7 (3): e182-84. https://doi.org/10.1093/jpids/piy064.

Fischer-Le Saux, M, V Viallard, B Brunel, Phillippe Normand, and N E Boemare. 1999. "Polyphasic Classification of the Genus Photorhabdus and Proposal of New Taxa: P. Luminescens Subsp. Luminescens Subsp. Nov., P. Luminescens Subsp. Akhurstii Subsp. Nov., P. Luminescens Subsp. Laumondii Subsp. Nov., P. Temperata Sp. Nov., P. Temperata Subs.” International Journal of Systematic Bacteriology 49 (4): 1645-56. https://doi.org/10.1099/00207713-49-4-1645.

Gerrard, John G, and Robert P Stevens. 2017. “A Review of Clinical Cases of Infection with Photorhabdus Asymbiotica." In The Molecular Biology of Photorhabdus Bacteria, edited by Richard H ffrench-Constant, 179-91. Cham: Springer International Publishing. https://doi.org/10.1007/82_2016_56.

Guijarro, José A., Desirée Cascales, Ana I. García-Torrico, Mario García-Domínguez, and Jessica Méndez. 2015. “Temperature-Dependent Expression of Virulence Genes in Fish-Pathogenic Bacteria." Frontiers in Microbiology 6 (JUL): 1-11. https://doi.org/10.3389/fmicb.2015.00700.

Hoang, Diep Thi, Olga Chernomor, Arndt von Haeseler, Bui Quang Minh, and Le Sy Vinh. 2018. "UFBoot2: Improving the Ultrafast Bootstrap Approximation." Molecular Biology and Evolution 35 (2): 518-22. https://doi.org/10.1093/molbev/msx281.

Ishioka, Ken, Hiroshi Iwasaki, and Hideo Shinagawa. 1997. "Roles of the RecG Gene Product of Escherichia Coli in Recombination Repair: Effects of the .DELTA.RecG Mutation on Cell Division and Chromosome Partition.” Genes \& Genetic Systems 72 (2): 91-99. https://doi.org/10.1266/ggs.72.91.

Jones, Robert T, Maria Sanchez-Contreras, Isabella Vlisidou, Matthew R Amos, Guowei Yang, Xavier Muñoz-Berbel, Abhishek Upadhyay, et al. 2010. "Photorhabdus Adhesion Modification Protein (Pam) Binds Extracellular Polysaccharide and Alters Bacterial Attachment.” BMC Microbiology 10 (1): 
681

682

683

684

685

686

687

688

689

690

691

692

693

694

695

696

697

698

699

700

701

702

703

704

705

706

707

708

141. https://doi.org/10.1186/1471-2180-10-141.

Kalyaanamoorthy, Subha, Bui Quang Minh, Thomas K F Wong, Arndt von Haeseler, and Lars S Jermiin. 2017. "ModelFinder: Fast Model Selection for Accurate Phylogenetic Estimates.” Nature Methods 14 (6): 587-89. https://doi.org/10.1038/nmeth.4285.

Kazimierczak, Waldemar, Henryk Skrzypek, Ewa Sajnaga, Marcin Skowronek, Adam Waśko, and Anna Kreft. 2017. "Strains of Photorhabdus Spp. Associated with Polish Heterorhabditis Isolates: Their Molecular and Phenotypic Characterization and Symbiont Exchange." Archives of Microbiology 199 (7): 979-89. https://doi.org/10.1007/s00203-017-1368-z.

Koboldt, Daniel C, Qunyuan Zhang, David E Larson, Dong Shen, Michael D Mclellan, Ling Lin, Christopher A Miller, Elaine R Mardis, Li Ding, and Richard K Wilson. 2012. "VarScan 2 : Somatic Mutation and Copy Number Alteration Discovery in Cancer by Exome Sequencing," 568-76. https://doi.org/10.1101/gr.129684.111.568.

Kumar, Gokhlesh, Simon Menanteau-Ledouble, Mona Saleh, and Mansour ElMatbouli. 2015. "Yersinia Ruckeri, the Causative Agent of Enteric Redmouth Disease in Fish." Veterinary Research 46 (1): 1-10. https://doi.org/10.1186/s13567-015-0238-4.

Lages, Marta A., Miguel Balado, and Manuel L. Lemos. 2019. “The Expression of Virulence Factors in Vibrio Anguillarum Is Dually Regulated by Iron Levels and Temperature." Frontiers in Microbiology 10 (October). https://doi.org/10.3389/fmicb.2019.02335.

Langmead, Ben, and Steven L Salzberg. 2012. "Fast Gapped-Read Alignment with Bowtie 2." Nat Methods 9 (4): 357-59. https://doi.org/10.1038/nmeth.1923.

Lango, Lea, and David J Clarke. 2010. “A Metabolic Switch Is Involved in Lifestyle Decisions in Photorhabdus Luminescens." Molecular Microbiology 77 (6): 1394-1405. https://doi.org/10.1111/j.1365-2958.2010.07300.x. 
on the DsrA Small Regulatory RNA.” Journal of Molecular Biology 344 (5): 1211-23. https://doi.org/10.1016/j.jmb.2004.10.006.

Letunic, Ivica, and Peer Bork. 2019. "Interactive Tree Of Life (ITOL) v4: Recent Updates and New Developments.” Nucleic Acids Research 47 (W1): W256-59. https://doi.org/10.1093/nar/gkz239.

Li, Heng, Bob Handsaker, Alec Wysoker, Tim Fennell, Jue Ruan, Nils Homer, Gabor Marth, Goncalo Abecasis, and Richard Durbin. 2009. "The Sequence Alignment/Map Format and SAMtools.” Bioinformatics 25 (16): 2078-79. https://doi.org/10.1093/bioinformatics/btp352.

Machado, Ricardo A. R., Daniel Wüthrich, Peter Kuhnert, Carla C. M. Arce, Lisa Thönen, Celia Ruiz, Xi Zhang, et al. 2018. "Whole-Genome-Based Revisit of Photorhabdus Phylogeny: Proposal for the Elevation of Most Photorhabdus Subspecies to the Species Level and Description of One Novel Species Photorhabdus Bodei Sp. Nov., and One Novel Subspecies Photorhabdus Laumondii Subsp. ." International Journal of Systematic and Evolutionary Microbiology 68 (8): 2664-81. https://doi.org/10.1099/ijsem.0.002820.

Maher, Abigail M D, Mohamed A M Asaiyah, Caroline Brophy, and Christine T Griffin. 2017. "An Entomopathogenic Nematode Extends Its Niche by Associating with Different Symbionts.” Microbial Ecology 73 (1): 211-23. https://doi.org/10.1007/s00248-016-0829-2.

Maneesakorn, Patchareewan, Ruisheng An, Hannah Daneshvar, Kara Taylor, Xiaodong Bai, Byron J. Adams, Parwinder S. Grewal, and Angsumarn Chandrapatya. 2011. "Phylogenetic and Cophylogenetic Relationships of Entomopathogenic Nematodes (Heterorhabditis: Rhabditida) and Their Symbiotic Bacteria (Photorhabdus: Enterobacteriaceae).” Molecular Phylogenetics and Evolution 59 (2): 271-80. https://doi.org/10.1016/j.ympev.2011.02.012.

Mathur, Chetna, Jyoti Kushwah, Vishal S. Somvanshi, and Tushar K. Dutta. 2018. "A 37 KDa Txp40 Protein Characterized from Photorhabdus Luminescens Sub Sp. Akhurstii Conferred Injectable and Oral Toxicity to Greater Wax Moth, Galleria 
740

741

742

743

744

Mellonella." Toxicon 154 (November): 69-73.

https://doi.org/10.1016/j.toxicon.2018.09.007.

Medema, Marnix H., Eriko Takano, and Rainer Breitling. 2013. "Detecting Sequence Homology at the Gene Cluster Level with Multigeneblast." Molecular Biology and Evolution 30 (5): 1218-23. https://doi.org/10.1093/molbev/mst025.

Mendez, Jessica, Desirée Cascales, Ana I Garcia-Torrico, and Jose A Guijarro. 2018. "Temperature-Dependent Gene Expression in Yersinia Ruckeri: Tracking Specific Genes by Bioluminescence During in Vivo Colonization." Frontiers in Microbiology 9: 1098. https://doi.org/10.3389/fmicb.2018.01098.

Møller, Thorleif, Thomas Franch, Peter Højrup, Douglas R Keene, Hans Peter Bächinger, Richard G Brennan, and Poul Valentin-Hansen. 2002. "Hfq: A Bacterial Sm-like Protein That Mediates RNA-RNA Interaction.” Molecular Cell 9 (1): 23-30. https://doi.org/10.1016/s1097-2765(01)00436-1.

Mulley, Geraldine, Michael L. Beeton, Paul Wilkinson, Isabella Vlisidou, Nina Ockendon-Powell, Alexia Hapeshi, Nick J. Tobias, et al. 2015. "From Insect to Man: Photorhabdus Sheds Light on the Emergence of Human Pathogenicity." Edited by Mikael Skurnik. PLOS ONE 10 (12): e0144937. https://doi.org/10.1371/journal.pone.0144937.

Nguyen, Lam Tung, Heiko A. Schmidt, Arndt Von Haeseler, and Bui Quang Minh. 2015. "IQ-TREE: A Fast and Effective Stochastic Algorithm for Estimating Maximum-Likelihood Phylogenies.” Molecular Biology and Evolution 32 (1): 268-74. https://doi.org/10.1093/molbev/msu300.

Pettersen, Eric F., Thomas D. Goddard, Conrad C. Huang, Gregory S. Couch, Daniel M. Greenblatt, Elaine C. Meng, and Thomas E. Ferrin. 2004. "UCSF Chimera A Visualization System for Exploratory Research and Analysis." Journal of Computational Chemistry. https://doi.org/10.1002/jcc.20084.

Philippe, Nadège, Jean Pierre Alcaraz, Evelyne Coursange, Johannes Geiselmann, and Dominique Schneider. 2004. "Improvement of PCVD442, a Suicide Plasmid for Gene Allele Exchange in Bacteria." Plasmid 51 (3): 246-55. 
https://doi.org/10.1016/j.plasmid.2004.02.003.

770

771

772

773

774

775

776

Rudolph, Christian J, Akeel a Mahdi, Amy L Upton, and Robert G Lloyd. 2010. "RecG Protein and Single-Strand DNA Exonucleases Avoid Cell Lethality Associated with PriA Helicase Activity in Escherichia Coli." Genetics 186 (2): 473-92. https://doi.org/10.1534/genetics.110.120691.

Sievers, Fabian, Andreas Wilm, David Dineen, Toby J Gibson, Kevin Karplus, Weizhong Li, Rodrigo Lopez, et al. 2011. "Fast, Scalable Generation of HighQuality Protein Multiple Sequence Alignments Using Clustal Omega,” no. 539. https://doi.org/10.1038/msb.2011.75.

Solovyev, Victor, and Asaf Salamov. 2011. "Automatic Annotation of Microbial Genomes and Metagenomic Sequences.” In Metagenomics and Its Applications in Agriculture, Biomedicine and Environmental Studies.

Somvanshi, V. S., R. E. Sloup, J. M. Crawford, A. R. Martin, A. J. Heidt, K.-s. Kim, J. Clardy, and T. A. Ciche. 2012. "A Single Promoter Inversion Switches Photorhabdus Between Pathogenic and Mutualistic States.” Science 337 (6090): 88-93. https://doi.org/10.1126/science.1216641.

Tailliez, P., C. Laroui, N. Ginibre, A. Paule, S. Pages, and N. Boemare. 2010. "Phylogeny of Photorhabdus and Xenorhabdus Based on Universally Conserved Protein-Coding Sequences and Implications for the Taxonomy of These Two Genera. Proposal of New Taxa: X. Vietnamensis Sp. Nov., P. Luminescens Subsp. Caribbeanensis Subsp. Nov., P. L.” INTERNATIONAL JOURNAL OF SYSTEMATIC AND EVOLUTIONARY MICROBIOLOGY 60 (8): 1921-37. https://doi.org/10.1099/ijs.0.014308-0.

Tobias, Nicholas J., Antje K. Heinrich, Helena Eresmann, Patrick R. Wright, Nick Neubacher, Rolf Backofen, and Helge B. Bode. 2017. "Photorhabdus-Nematode Symbiosis Is Dependent on Hfq-Mediated Regulation of Secondary Metabolites." Environmental Microbiology 19 (1): 119-29. https://doi.org/10.1111/1462-2920.13502.

797 Vernikos, Georgios S, and Julian Parkhill. 2006. "Interpolated Variable Order Motifs 
798

799

800

801

802

803

804

805

806

807

808

809

810

811

812

813

814

815

816

for Identification of Horizontally Acquired DNA: Revisiting the Salmonella Pathogenicity Islands.” Bioinformatics (Oxford, England) 22 (18): 2196-2203. https://doi.org/10.1093/bioinformatics/btl369.

Waterfield, Nick R., Todd Ciche, and David Clarke. 2009. "Photorhabdus and a Host of Hosts." Annual Review of Microbiology 63 (1): 557-74. https://doi.org/10.1146/annurev.micro.091208.073507.

Zamora-Lagos, Maria-Antonia, Simone Eckstein, Angela Langer, Athanasios Gazanis, Friedhelm Pfeiffer, Bianca Habermann, and Ralf Heermann. 2018. "Phenotypic and Genomic Comparison of Photorhabdus Luminescens Subsp. Laumondii TT01 and a Widely Used Rifampicin-Resistant Photorhabdus Luminescens Laboratory Strain.” BMC Genomics 19 (1): 854. https://doi.org/10.1186/s12864-018-5121-z.

Zhang, A., S Altuvia, A Tiwari, L Argaman, R Hengge-Aronis, and G Storz. 1998. “The OxyS Regulatory RNA Represses RpoS Translation and Binds the Hfq (HF-I) Protein.” The EMBO Journal 17 (20): 6061-68. https://doi.org/10.1093/emboj/17.20.6061.

Zhang, Yang. 2008. "I-TASSER Server for Protein 3D Structure Prediction.” BMC Bioinformatics. https://doi.org/10.1186/1471-2105-9-40. 
Figure 1

A

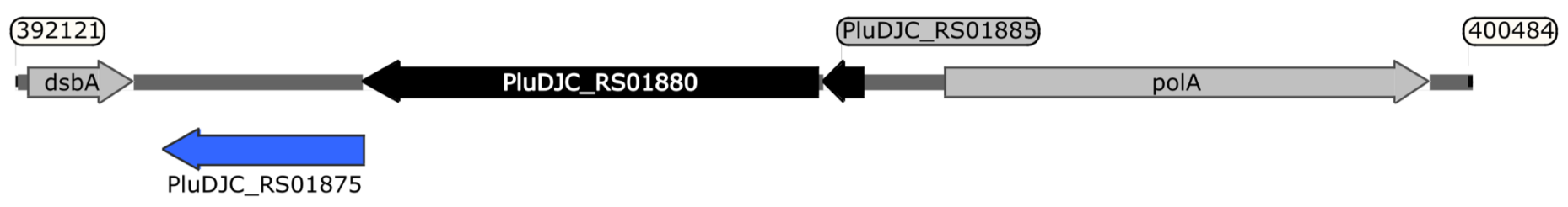

B N-terminus

C-terminus

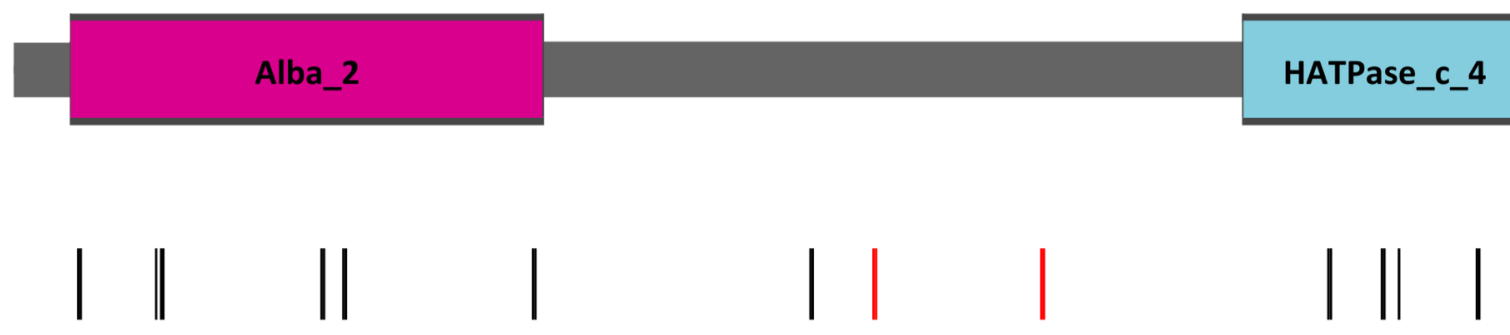

C

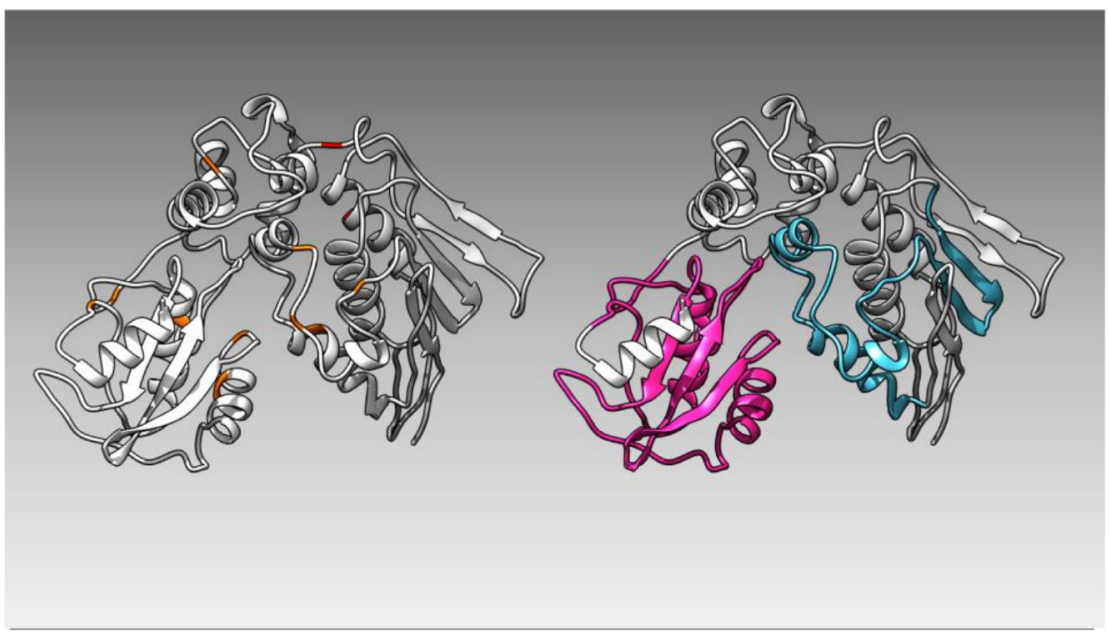


Figure 2

A

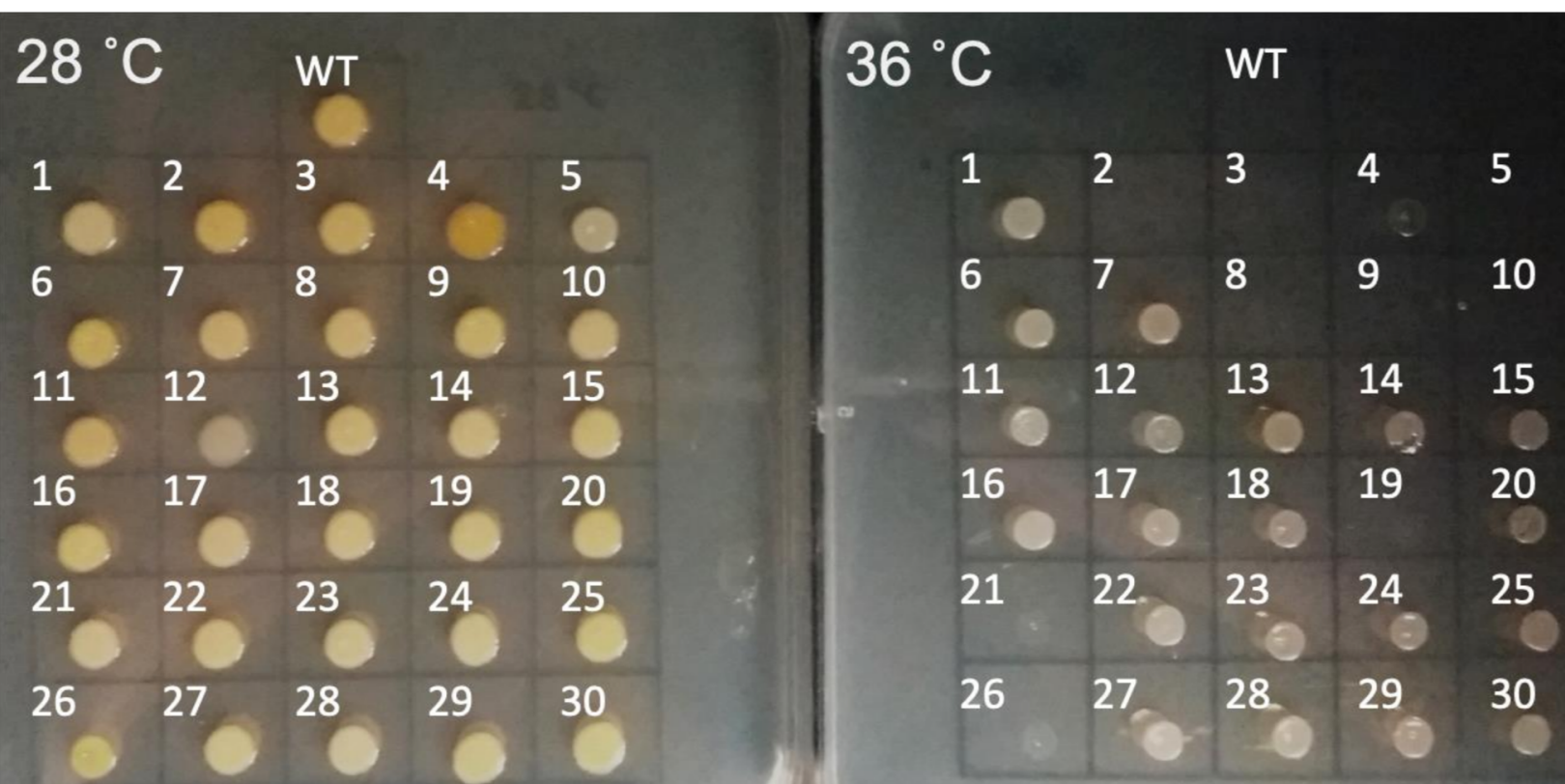

B

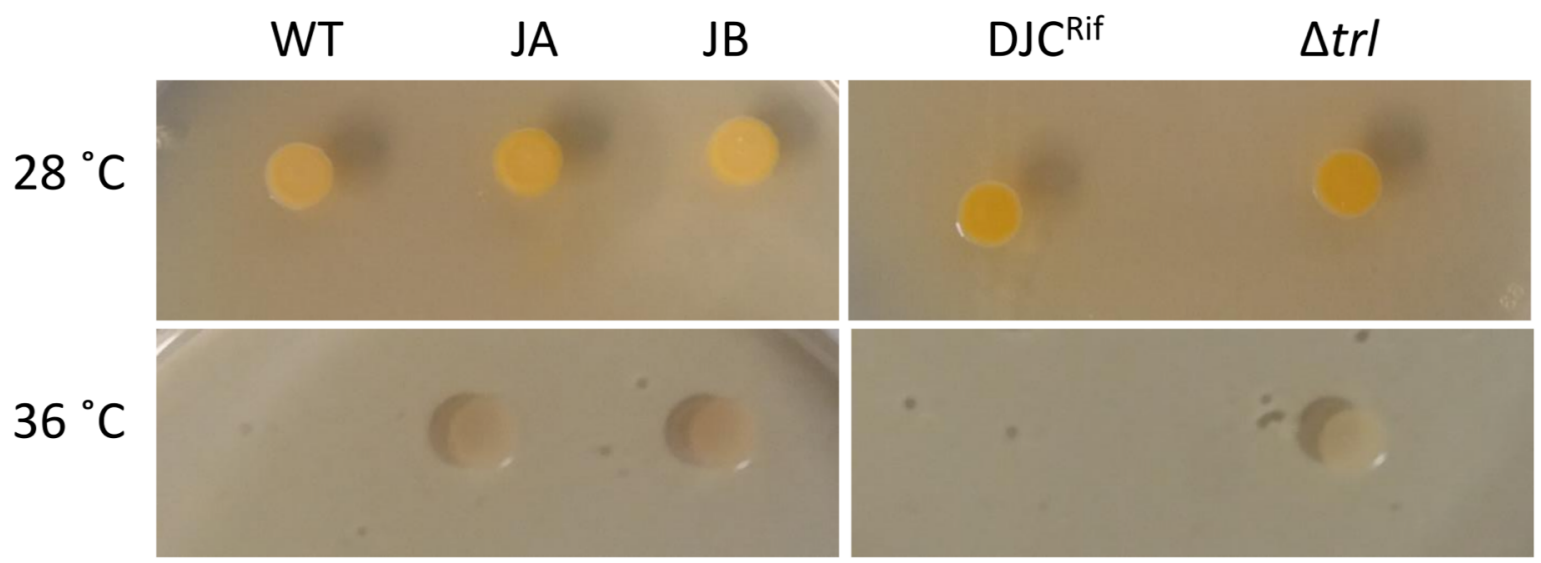


Figure 3

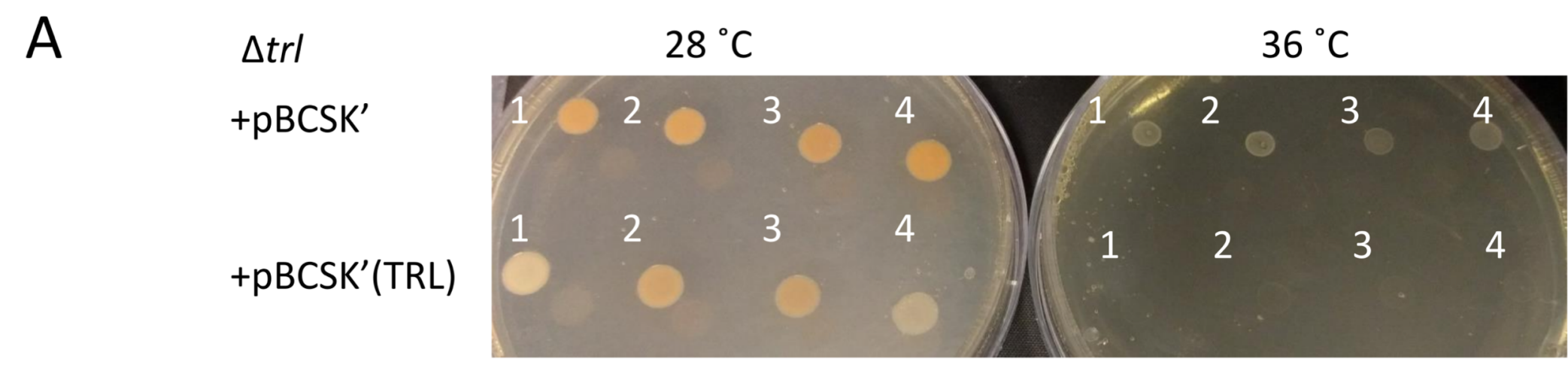

B

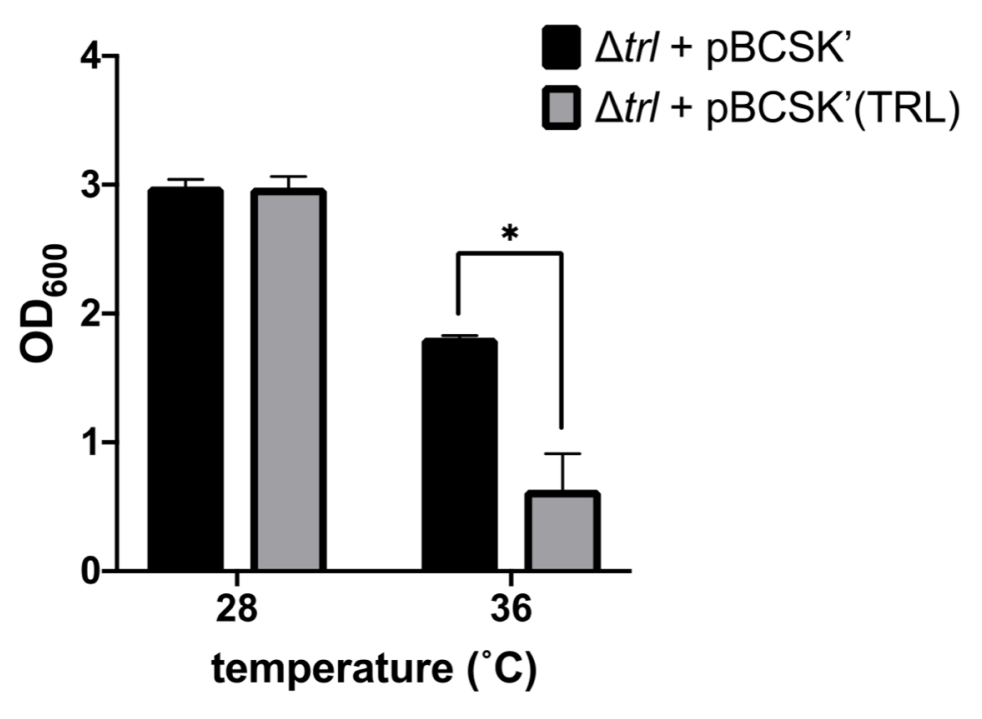


Figure 4

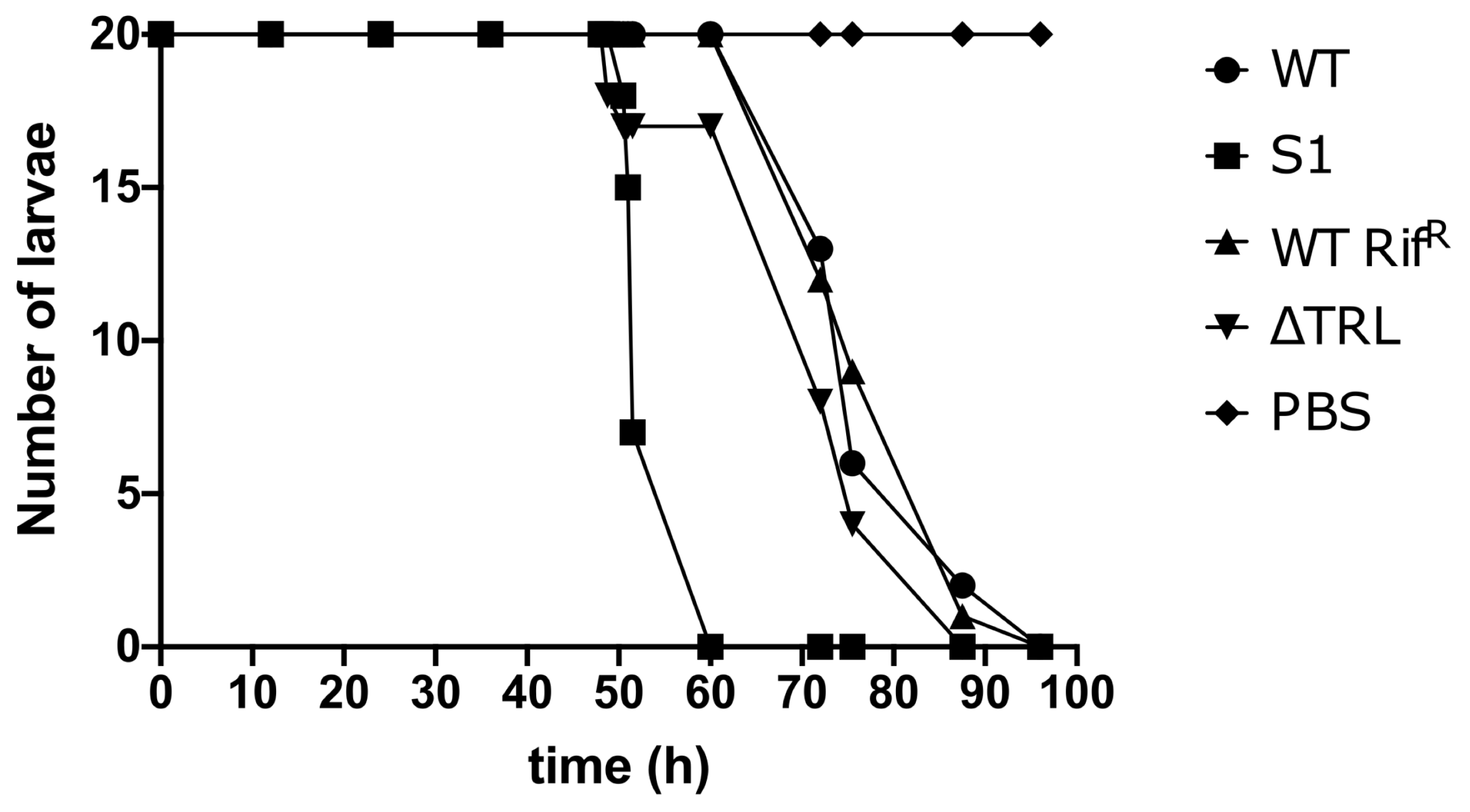


Figure 5

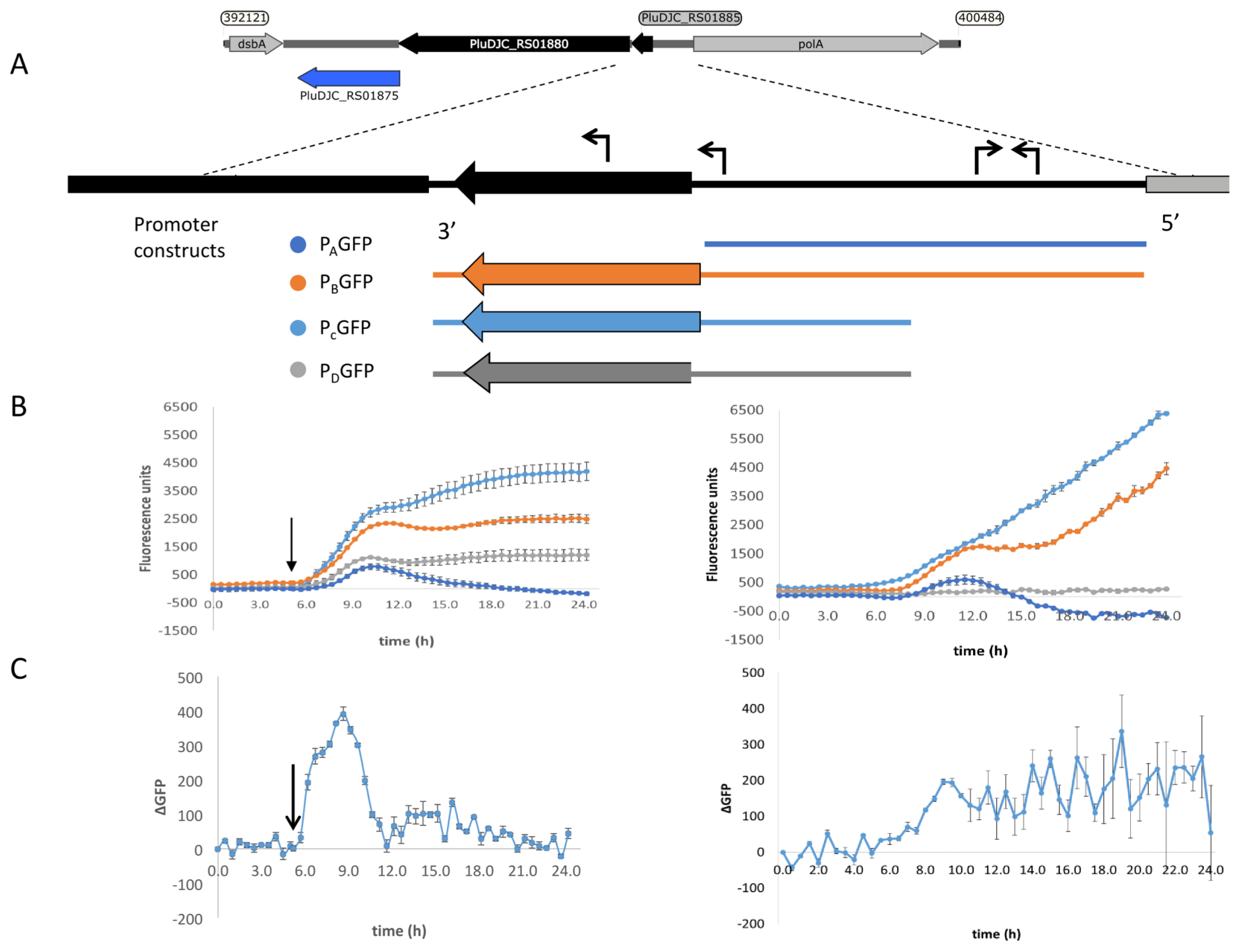




\section{Figure 6}

Tree scale: 0.1

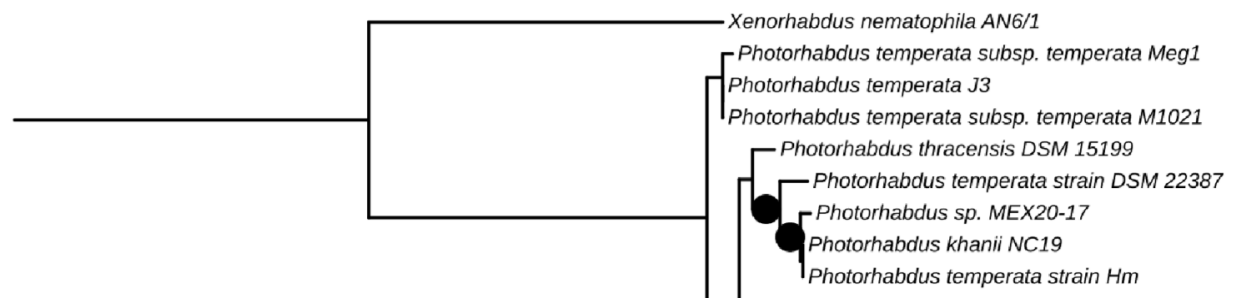

Photorhabdus temperata strain $\mathrm{Hm}$

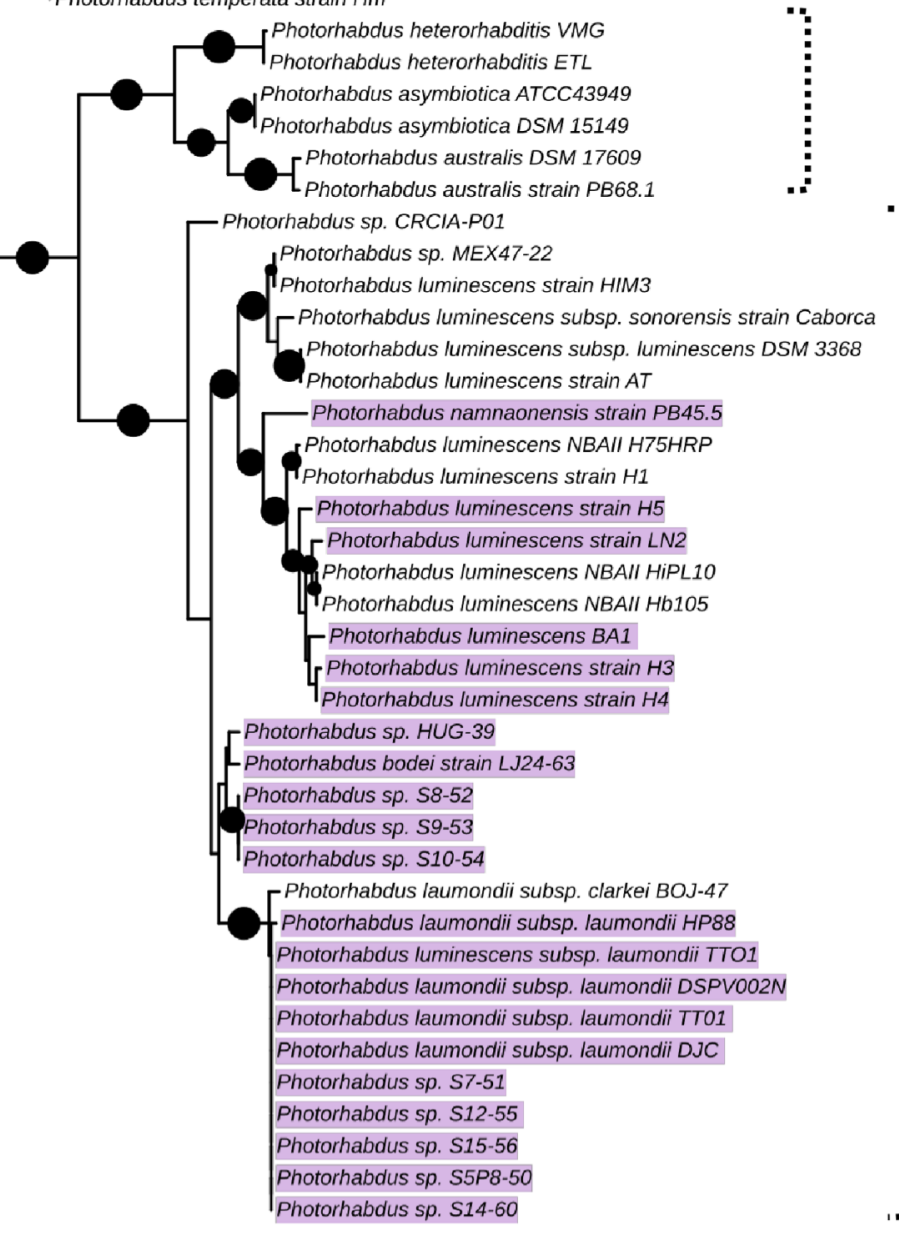

Temperata

subgroup

Asymbiotica

subgroup

\author{
Luminescens \\ subgroup
}


Figure 7

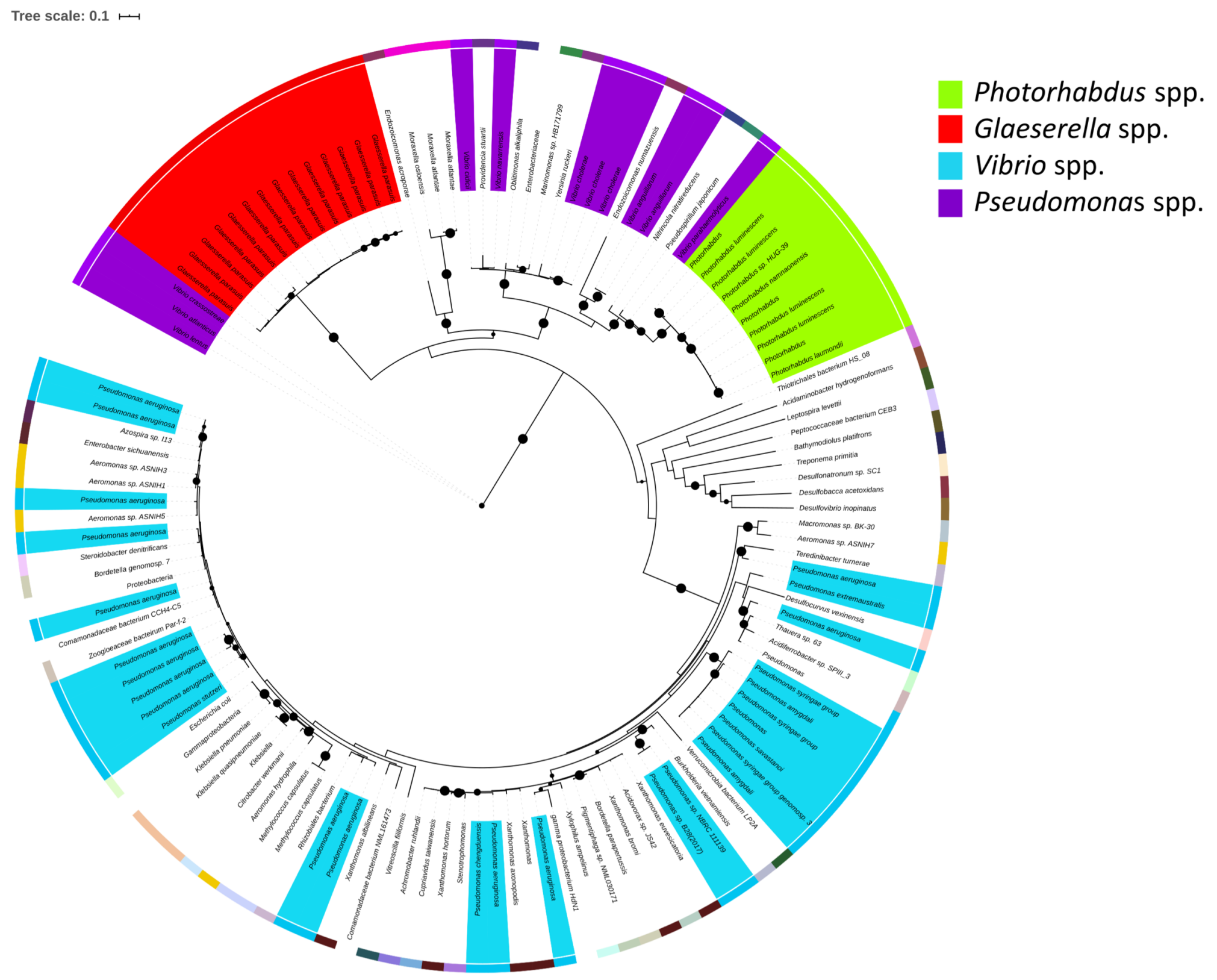




\section{Figure 8}

\section{Photorhabdus laumondii DJC}

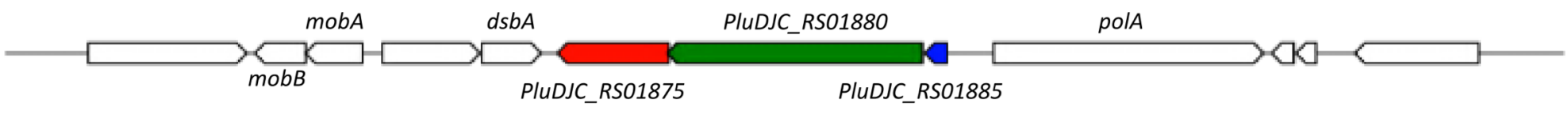

\section{Photorhabdus laumondii TTO1}

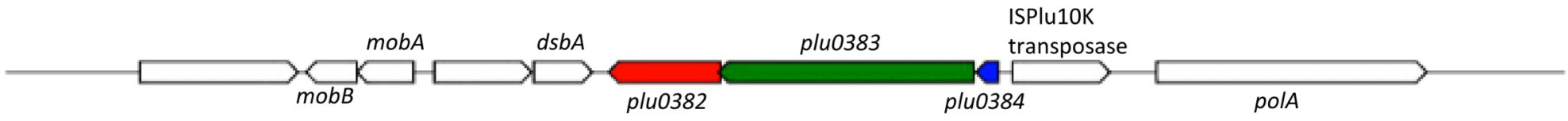

Vibrio anguillarum VIB43

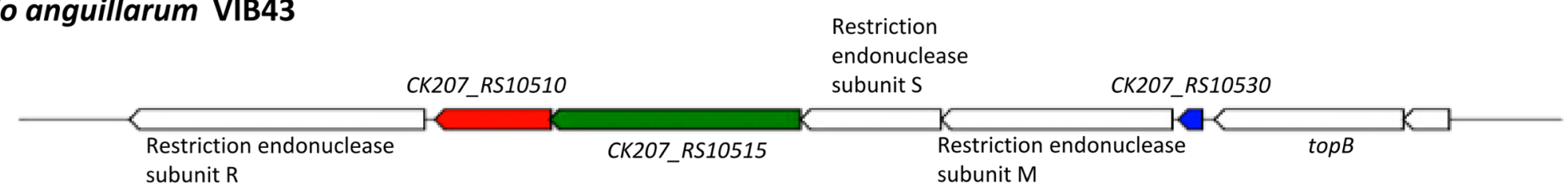

Yersinia ruckeri PB-H2, plasmid : pYR3.

DNA
mobile
protein

\section{Oblitimonas alkaliphila D2441}

$\begin{aligned} & \text { Restriction endonuclease } \\ & \text { subunit } \mathrm{R}\end{aligned}$
metal dependent
hydrolase

